\title{
BMJ Open Characteristics associated with COVID-19 vaccine uptake among adults aged 50 years and above in England (8 December 2020-17 May 2021): a population-level observational study
}

Elise Tessier (10 , ${ }^{1}$ Yuma Rai, ${ }^{1}$ Eleanor Clarke, ${ }^{1}$ Anissa Lakhani, ${ }^{1}$ Camille Tsang, ${ }^{1}$ Ashley Makwana, ${ }^{2}$ Heather Heard ${ }^{3}$ Tim Rickeard, ${ }^{2}$ Shreya Lakhani, ${ }^{1}$ Partho Roy, ${ }^{1}$ Michael Edelstein (1) ," Mary Ramsay (1) ," Jamie Lopez-Bernal (1) ,1 Joanne White, ${ }^{1}$ Nick Andrews (D) , ${ }^{1}$ Colin N J Campbell, ${ }^{1}$ Julia Stowe (D) ${ }^{1}$

To cite: Tessier E, Rai Y, Clarke E, et al. Characteristics associated with COVID-19 vaccine uptake among adults aged 50 years and above in England (8 December 2020-17 May 2021): a population-level observational study. BMJ Open 2022;12:e055278. doi:10.1136/ bmjopen-2021-055278

- Prepublication history and additional supplemental material for this paper are available online. To view these files, please visit the journal online (http://dx.doi.org/10.1136/ bmjopen-2021-055278).

Received 08 July 2021 Accepted 07 February 2022
Check for updates

(C) Author(s) (or their employer(s)) 2022. Re-use permitted under CC BY-NC. No commercial re-use. See rights and permissions. Published by BMJ.

For numbered affiliations see end of article.

Correspondence to

Ms Elise Tessier;

elise.tessier@phe.gov.uk

\section{ABSTRACT}

Objective To determine characteristics associated with COVID-19 vaccine coverage among individuals aged 50 years and above in England since the beginning of the programme.

Design Observational cross-sectional study assessed by logistic regression and mean prevalence margins.

Setting COVID-19 vaccinations delivered in England from 8 December 2020 to 17 May 2021.

Participants 30624 257/61 967781 (49.4\%) and 17

360 045/61 967781 (28.1\%) individuals in England were recorded as vaccinated in the National Immunisation Management System with a first dose and a second dose of a COVID-19 vaccine, respectively.

Interventions Vaccination status with COVID-19 vaccinations.

Main outcome measures Proportion, adjusted ORs and mean prevalence margins for individuals not vaccinated with dose 1 among those aged 50-69 years and dose 1 and 2 among those aged 70 years and above.

Results Of individuals aged 50 years and above, black/ African/Caribbean ethnic group was the least likely of all ethnic groups to be vaccinated with dose 1 of the COVID-19 vaccine. However, of those aged 70 years and above, the odds of not having dose 2 was 5.53 (95\% Cl 5.42 to 5.63) and 5.36 (95\% Cl 5.29 to 5.43 ) greater among Pakistani and black/African/Caribbean compared with white British ethnicity, respectively. The odds of not receiving dose 2 was $1.18(95 \% \mathrm{Cl} 1.16$ to 1.20) higher among individuals who lived in a care home compared with those who did not. This was the opposite to that observed for dose 1, where the odds of being unvaccinated was significantly higher among those not living in a care home $(0.89$ (95\% $\mathrm{Cl} 0.87$ to 0.91$))$. Conclusions We found that there are characteristics associated with low COVID-19 vaccine coverage. Inequalities, such as ethnicity are a major contributor to suboptimal coverage and tailored interventions are required to improve coverage and protect the population from SARS-CoV-2.

\section{Strengths and limitations of this study}

- This is the first study assessing characteristics associated with COVID-19 vaccine coverage for all individuals aged 50 years and above in England.

- This study uses data from the National Immunisation Management System, which is based on all individuals in England with a registered National Health Service (NHS) number.

- This centralised national system captures individuallevel data for both vaccination status and demographic characteristics and allows for linkage to other datasets such as healthcare worker and care home resident status.

- This study does not include those without an NHS number and, therefore, it is possible we have underestimated the number of vaccines delivered and odds of being unvaccinated for characteristics such as ethnic groups where we have seen the greatest impact.

- Residual errors in data entry on the point-of-care applications at the vaccination sites may have also occurred, although these errors are not likely to be widespread.

\section{BACKGROUND}

The UK was the world's first country to approve a COVID-19 vaccine for the pandemic, getting a head start on the roll out of its COVID-19 vaccination programme. ${ }^{1}$ On 8 December 2020, the UK launched its COVID-19 vaccination programme with the aim of reducing COVID-19 mortality and hospitalisations among those at highest risk. There are currently three vaccines that the Medicines and Healthcare products Regulatory Agency have authorised: the Pfizer/ BioNTech vaccine (offered from 8 December 2020), the AstraZeneca (Oxford) vaccine 
(offered from 4 January 2021) and the Moderna Tx (offered from 13 April 2021). ${ }^{2-4}$

To ensure the reduction of mortality from SARS-CoV-2 infection and to protect the healthcare system, the Joint Committee on Vaccination and Immunisation (JCVI), an independent expert advisory committee that advises UK health departments on vaccination, initially recommended extending the interval between doses to as long as 12 weeks. Its goal was to vaccinate a greater number of people sooner with the first dose of the COVID-19 vaccine. ${ }^{56}$ The JCVI also recommended that the vaccination programme be rolled out in phases beginning on 8 December 2021, for those aged 80 years and above and for frontline healthcare workers. ${ }^{6}$ The UK then opened eligibility to others, first among individuals identified as clinically extremely vulnerable, meaning they had been classified as at risk and advised to shield at home, and then in descending age groups. ${ }^{7}$ By 17 March 2021, all adults aged 50 years and above were eligible for their first dose of a COVID-19 vaccine.

NHS England, with support from Public Health England (PHE), the national public health agency, publishes COVID-19 vaccination counts and denominators by region, age, ethnicity and for care home residents using a tracker tool. ${ }^{8}$ Previous research on characteristics for low vaccine coverage in England have shown that deprivation and ethnicity are associated with lower coverage for a range of routine immunisations delivered in England. ${ }^{9-11}$ There is little to no data collected on vaccine coverage for routine vaccines such as shingles and influenza for individuals living in care homes, although evidence shows that barriers exist in achieving high influenza uptake in care homes. These barriers include care home size, geographical location and working relationships with primary care and pharmacies. ${ }^{12}$ These studies and guidance assess routine vaccines delivered in England, which are primarily delivered through general practices, pharmacies and schools. With the ongoing COVID-19 pandemic, the rapid development of COVID-19 vaccines and the urgency to rapidly roll out the programme in various settings including mass immunisation sites, it is unknown whether the same characteristics associated with vaccine coverage for routine programmes are associated with low coverage for COVID-19 vaccines.

The aim of this study is twofold:

1. To describe the number of individuals eligible for a COVID-19 vaccine in the first phase of the roll out that have been vaccinated with a single or two doses of COVID-19 vaccine by age, sex, geographical location, vaccine type, ethnicity, deprivation, urban or rural setting and programme week.

2. To determine whether there are any characteristics independently associated with low vaccine coverage for dose 1 and dose 2 of COVID-19 vaccines.

\section{METHODS}

\section{Data source}

A National Immunisation Management System (NIMS) capable of recording any vaccination, regardless of point of delivery, has been used for the pandemic response to collect information about COVID-19 vaccines delivered across England. Individuals who present to a vaccination site, such as general practice, pharmacy or hospital provider and receive a COVID-19 vaccine will have their vaccine event information recorded on a point-of-care application. PHE receives data that are linked to demographic data obtained from the National Health Service (NHS) (eg, gender, date of birth), using the individual's unique NHS number. ${ }^{13}$ PHE uses these data for monitoring vaccine safety, effectiveness and coverage. All variables for vaccine coverage are described in online supplemental table 1. Age was calculated for all individuals based on their age on 31 March 2021.

\section{Patient and public involvement}

No patients were involved in the design or execution of the study.

\section{Study population}

NIMS data were extracted on 17 May 2021 to assess vaccination status of all individuals aged 50-69 years for a first dose of COVID-19 vaccine, and of all individuals aged 70 years and above vaccinated with a first and a second dose of a COVID-19 vaccine. The programme was still being rolled out among younger cohorts, so not all aged between 50 and 59 years had been offered a second dose of a COVID-19 vaccine at the time of data extraction. Individuals recorded in the NIMS must have an NHS number in order to link the population denominator and vaccination event files. All individuals with a death recorded were excluded from the analyses for the purpose of calculating coverage in the living resident population aged 50 years and above on 31 March 2021.

\section{Vaccine coverage}

Vaccine coverage was calculated by dividing the total number of individuals with a recorded NHS number in the dataset who were vaccinated with one dose and two doses of COVID-19 vaccine since the beginning of the vaccine roll out on 8 December 2020 (numerator) by the total number of individuals with a recorded NHS number in the dataset (denominator).

\section{Proportion of unvaccinated individuals}

The proportion of individuals not vaccinated for dose 1 and/or not vaccinated with dose 2 was calculated by dividing the total number of individuals unvaccinated or with a single dose of the COVID-19 vaccine since the beginning of the vaccine roll out on 8 December 2020 (numerator) by the total number of individuals with a recorded NHS number in the dataset (denominator).

\section{Descriptive analyses}

The proportion of individuals vaccinated and unvaccinated was described by programme week, starting at week 1 (beginning on 7 December 2020). Cumulative vaccine uptake has been calculated by programme week. Furthermore, the number of and proportion of individuals 
unvaccinated were aggregated by age groups, region, rural/urban classification, ethnicity, for individuals clinically extremely vulnerable, for individuals over 65 years living in a care home, for individuals $<65$ years and a healthcare worker.

\section{Statistical analyses}

Statistical analyses were conducted for individuals eligible for a COVID-19 vaccine in England in phase I, at the beginning of the roll out. The analyses were conducted for those vaccinated with dose 1 and dose 2 for individuals aged 70 years and above, and for dose 1 among individuals aged $50-69$ years.

To assess the odds of being unvaccinated or being unvaccinated with dose 2 , univariable logistic regression models for each characteristic were fitted with the binary outcome of vaccination status (not vaccinated/vaccinated with dose 1 and vaccinated with dose 1 /vaccinated with dose 2). A multivariable logistic regression model was conducted adjusting for all other characteristics.

The adjusted mean prevalence margins and 95\% CIs of being unvaccinated for each characteristic within the multivariable model fit were calculated using the adjusted ratios. All statistical analyses were conducted using Stata V.15.1.

\section{RESULTS}

\section{Descriptive results}

From 17 March 2021, all individual aged 50 years and above were eligible for a COVID-19 vaccine. As of 17 May 2021, a total of $30624257 / 61967781(49.4 \%)$ and 17 360 045/61 967781 (28.1\%) individuals were vaccinated with a first dose and a second dose of a COVID-19 vaccine, respectively. A total of $90.9 \%$ of individuals aged 65 years and older had received at least one dose of the COVID-19 vaccine. The number of individuals vaccinated varied by programme week, plateauing at weeks 11 and 24 for dose 1 and 2 among those aged 70 years and above, and week 23 for dose 1 among those aged 50-69 years (figures 1

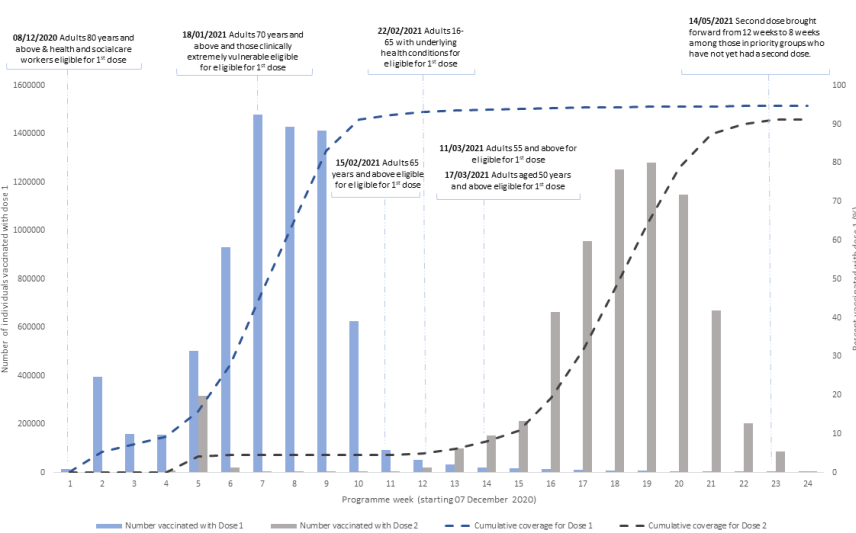

Figure 1 Number of individuals aged 70 years and above vaccinated with dose 1 and dose 2 of the COVID-19 vaccine and cumulative vaccine uptake by programme week (starting the week of 7 December 2020), England.

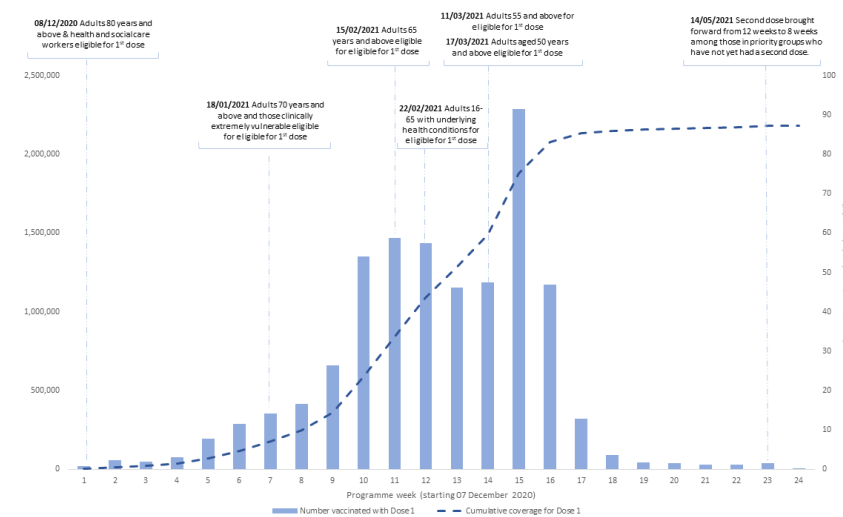

Figure 2 Number of individuals aged 50-69 years vaccinated with dose 1 of the COVID-19 vaccine and cumulative vaccine uptake by programme week (starting the week of 7 December 2020), England.

and 2). Those aged 50-69 years were still eligible for their second dose and had not yet plateaued at the time the data were extracted.

The proportion of individuals unvaccinated varied by age, dose and region; London consistently had the highest proportion of individuals being unvaccinated (figure 3). Among the total population aged 50 years and older, the North East of England had the lowest proportion of unvaccinated individuals (figure 3).

\section{Individuals aged $\mathbf{7 0}$ years and above unvaccinated}

Of individuals aged 70 years and above, all characteristics were significantly associated with being unvaccinated in both the univariable and multivariable logistic regression analyses.

The odds of being unvaccinated was higher among male population and those aged 75-79 and 80 years and above than the baseline of 70-74 years old (table 1). The odds of being unvaccinated with one or two doses was higher in urban areas, particularly in London with an increased odds of 2.30 (95\% CI 2.27 to 2.33) and 1.96 (95\% CI 1.94
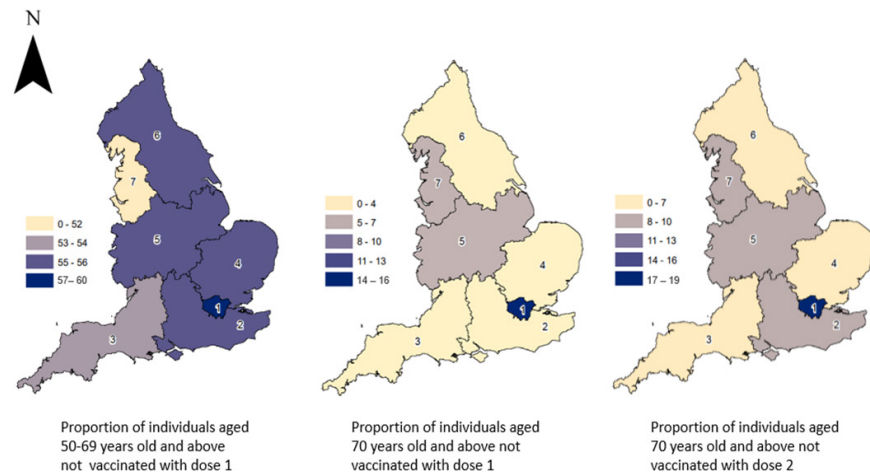

Figure 3 Proportion of individuals aged (A) 50-69 years not vaccinated with dose 1, (B) 70 years and above not vaccinated with dose 1 and (C) 70 years and above not vaccinated with dose 2 of COVID-19 vaccine (1: London; 2 : South East; 3: South West; 4: East of England; 5: Midlands; 6: North East and Yorkshire; 7: North West). 


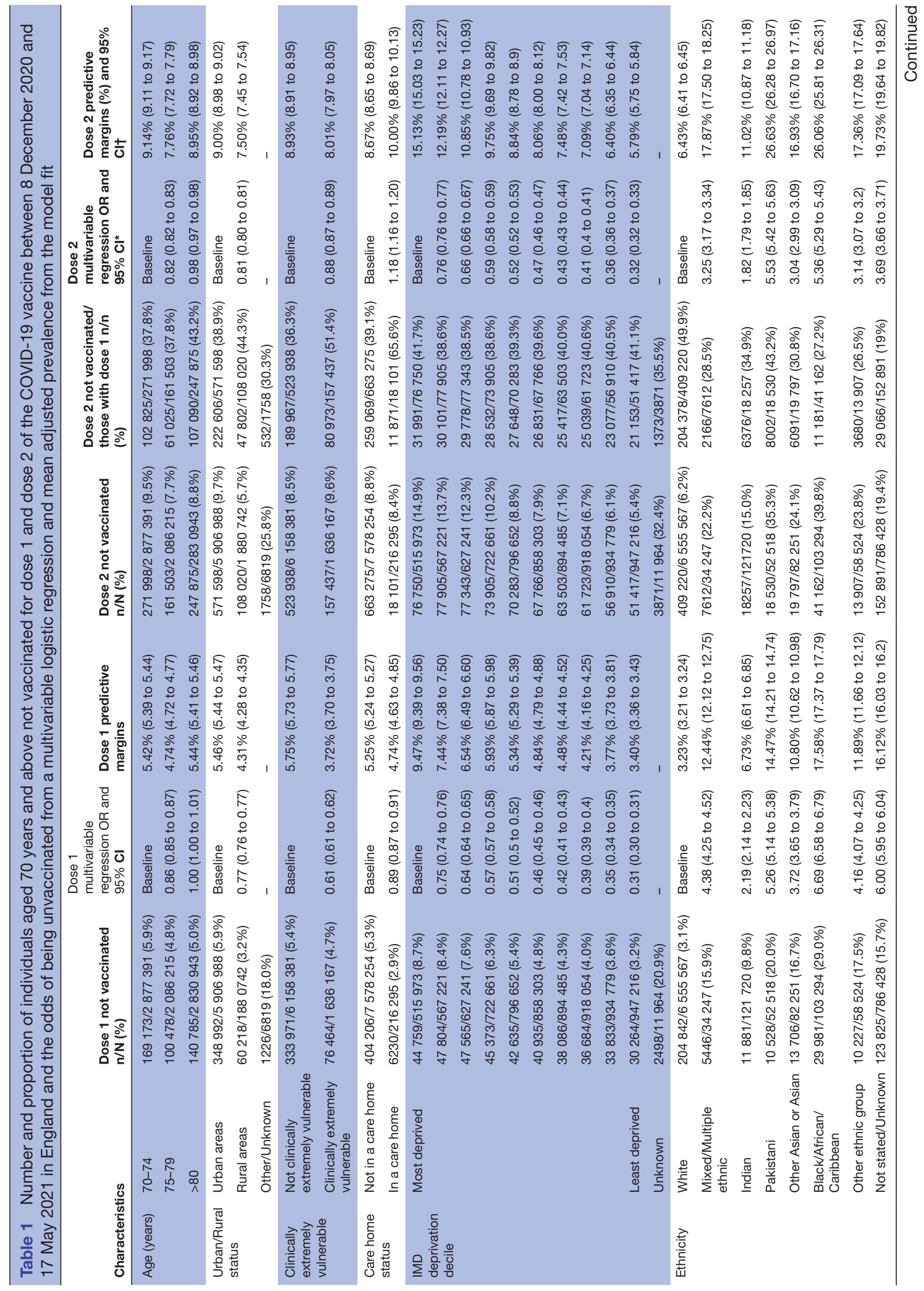




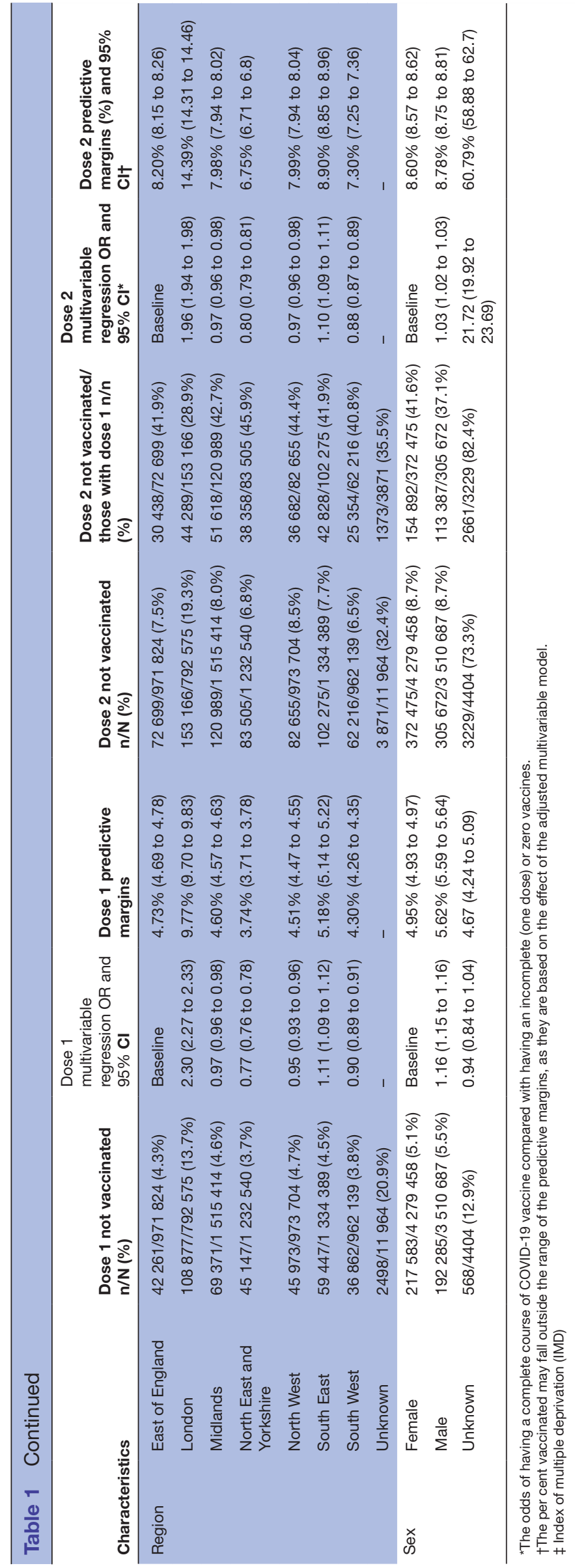

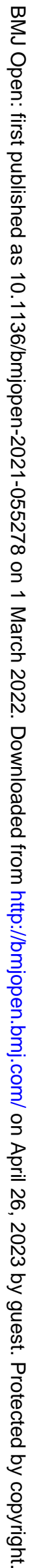


to 1.98) odds for being unvaccinated for both dose 1 and dose 2 , respectively (table 1 ).

Among those clinically extremely vulnerable, the odds of being unvaccinated was reduced (OR $0.61 \quad(95 \%$ CI 0.61 to 0.62$)$ ) and (OR 0.88 (95\% CI 0.87 to 0.89$)$ ) for dose 1 and dose 2, respectively compared with those with no significant underlying health conditions.

The odds of being unvaccinated among those living in a care home was also reduced for dose 1 (OR 0.89 (95\% CI 0.87 to 0.91$)$ ) compared with those not living in a care home. However, the odds of being unvaccinated for dose 2 was 1.18 (95\% CI 1.16 to 1.20 ) greater.

The greatest odds of being unvaccinated among those aged 70 years was among those living in the most deprived areas and among black/African/Caribbean ethnicities. Among the black/African/Caribbean ethnicity, the odds of not having a first dose was 6.69 (95\% CI 6.58 to 6.79) greater than white British ethnicity. The odds of being unvaccinated with a second dose was 5.53 (95\% CI 5.42 to 5.63) greater among the Pakistani ethnicity, followed by the black/African/Caribbean ethnicity with an odds of 5.36 (95\% CI 5.29 to 5.43$)$.

The mean prevalence of being unvaccinated for each characteristic within the adjusted multivariable model showed increased prevalence among the characteristics with the highest odds of being unvaccinated. The highest prevalence was among the black/African/Caribbean ethnic group for dose 1 with a mean prevalence of $17.6 \%$ unvaccinated. The prevalence among those unvaccinated with dose 1 was $26.6 \%$ and $26.1 \%$ among the Pakistani and black/African/Caribbean ethnicities, although the 95\% CIs do overlap between the two ethnicities (table 1).

\section{Individuals aged 50-69 years unvaccinated}

Of those aged 50-69 years, all the characteristics were significantly associated with being unvaccinated in both the univariable and multivariable logistic regression analyses.

Similarly to those aged 70 years and above, the odds of being unvaccinated was higher among male population and increased in the younger population (table 1). The odds of being unvaccinated was higher in urban areas and particularly in London where there was an increased odds of 1.85 (95\% CI 1.84 to 1.86) (table 2).

The greatest odds of being unvaccinated with dose 1 among 50-69 years age group was highest among those with an unknown or not stated ethnicity and black/ African/Caribbean ethnicity which had a 3.40 (95\% CI 3.38 to 3.41 ) and 3.32 (95\% CI 3.29 to 3.34) increased odds of being unvaccinated compared with those of white British ethnicity (table 2 ).

Both the clinically extremely vulnerable/at-risk and healthcare workers had decreased odds of being unvaccinated than those that were not in these groups (OR 0.59 (95\% CI 0.59 to 0.59 ) and 0.34 (95\% CI 0.34 to 0.35 ), respectively) (table 2), showing a protective effect among those at highest risk of SARS-CoV-2 disease.
The highest predictive margin for being unvaccinated after adjusting for all variables in the multivariable logistic regression model was among the unknown/not stated and black/African/Caribbean ethnic groups with a prevalence of $24.7 \%$ and $23.9 \%$ unvaccinated, respectively (table 2).

\section{DISCUSSION}

As of 17 May 2021, 49.4\% of the population aged 50 years and above in England had received a first dose of the COVID-19 vaccine and $28.1 \%$ had their second dose. Vaccine coverage for dose 1 among those aged 50 years, and for dose 2 for those aged 70 years and above, have plateaued. A significant increase in the number of individuals aged 50-69 years occurred in week 15 of the programme (the week commencing the 15 March 2021), when those aged 50-69 years became eligible for the vaccine. ${ }^{1415}$ Individuals aged 50-69 years vaccinated in earlier weeks were most likely to have been healthcare workers, individuals clinically extremely vulnerable/ cohort 6 at risk for SARS-CoV-2 disease. ${ }^{16}$

The overall proportion of individuals eligible for the vaccine but not vaccinated was highest in London, followed by the Midlands and East of England areas. These results coincide with findings from studies assessing predictors associated with influenza and shingles vaccine coverage, prior to mass immunisation, ${ }^{9} 1017$ which offered vaccines to individuals in similar populations, such as elderly individuals and those at risk, in which lower coverage in London and in urban areas is observed, despite differences in delivery of the programmes. For example, the shingles vaccine is delivered opportunistically in GP practices, while influenza vaccines are more widely available, including in pharmacies. Overall vaccine coverage for the first dose of a COVID-19 vaccine among those aged 65 years and older was higher for COVID-19 than for seasonal influenza vaccines delivered in the 2020/2021 winter season $(90.9 \%$ vs $80.9 \%) .^{18}$

Results from the multivariable logistic regression model and the predictive margins for two doses among those aged 70 years and above, and for one dose among those aged 50-69 years, indicate increased odds and predicted margins of being unvaccinated with lower age groups. Should lower coverage among younger adults continue to be observed as the programme continues to roll out, efforts must be made to address this. Moreover, after adjusting for all variables in the multivariable models, the odds of being unvaccinated was higher among male compared with female population, in urban areas compared with rural areas and highest in London compared with other regions in England among individuals aged 50 years and above. Reduced odds of being unvaccinated was observed among those clinically extremely vulnerable/at risk aged 50 years and above, and among healthcare workers aged 50-69 years. These individuals were all eligible for the vaccine early in the programme. 
Table 2 Number and proportion of individuals aged 50-69 years not vaccinated for dose 1 of the COVID-19 vaccine between 8 December 2020 and 17 May 2021 in England and the odds of being unvaccinated from a multivariable logistic regression and mean adjusted prevalence from the model fit

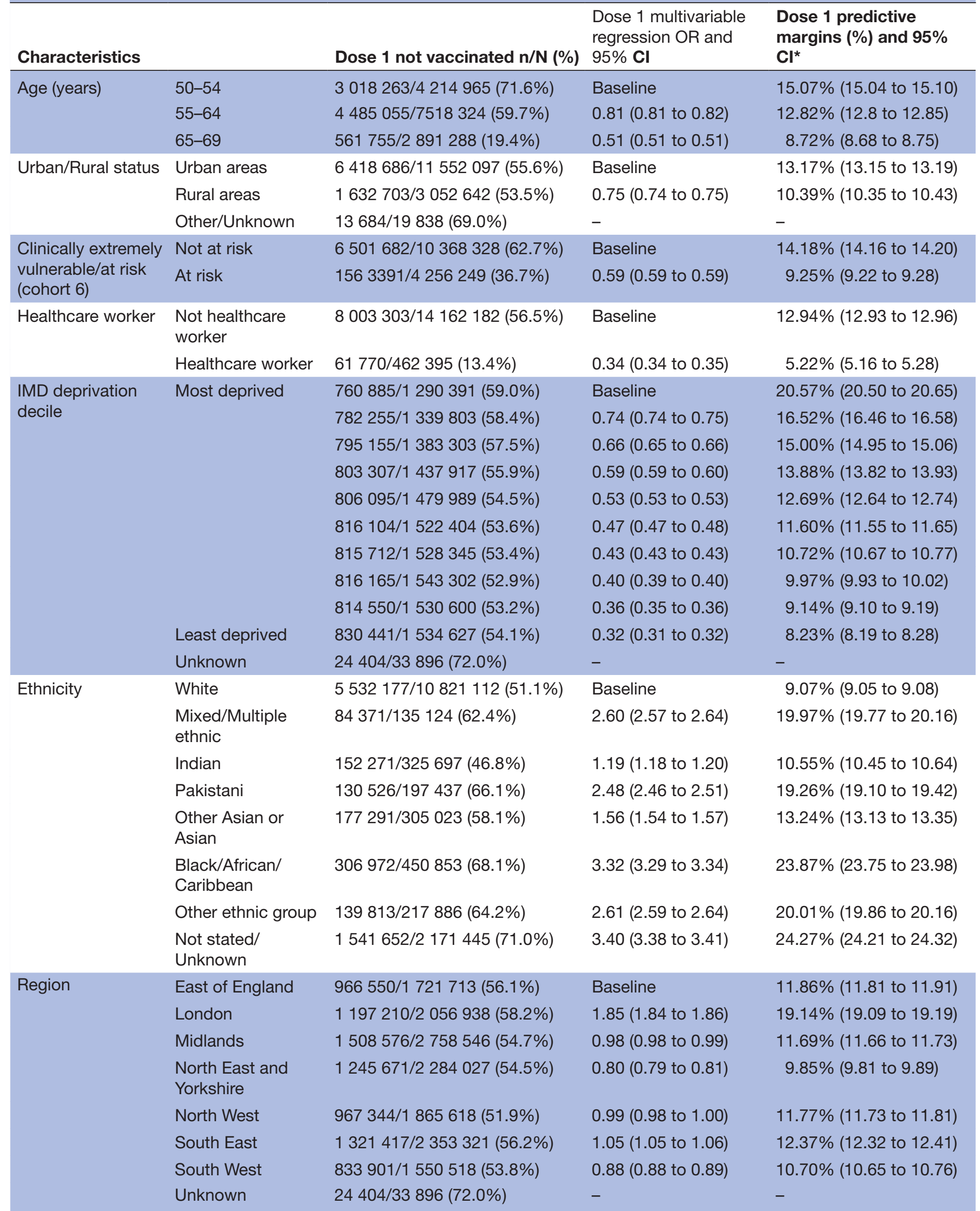




\begin{tabular}{|c|c|c|c|c|}
\hline Characteristics & & Dose 1 not vaccinated $n / N(\%)$ & $\begin{array}{l}\text { Dose } 1 \text { multivariable } \\
\text { regression OR and } \\
95 \% \mathbf{C l}\end{array}$ & $\begin{array}{l}\text { Dose } 1 \text { predictive } \\
\text { margins (\%) and } 95 \% \\
\mathrm{Cl}^{*}\end{array}$ \\
\hline \multirow{2}{*}{ Sex } & Male & 4388 976/7 376483 (59.5\%) & $1.27(1.27$ to 1.28$)$ & $13.87 \%(13.84$ to 13.89$)$ \\
\hline & Unknown & 16 169/19 031 (85.0\%) & $0.78(0.75$ to 0.81$)$ & $9.33 \%(9.01$ to 9.65$)$ \\
\hline
\end{tabular}

${ }^{*}$ The per cent vaccinated may fall outside the range of the predictive margins, as they are based on the effect of the adjusted multivariable model.

Individuals aged 70 years and above living in care homes had reduced odds of being unvaccinated for dose 1 compared with those not living in a care home. In contrast, care home residents had increased odds of being unvaccinated for the second dose compared with those living in a care home. The delivery of COVID-19 vaccines to care homes was primarily carried out by mobile teams that required a lot of logistics and coordination. ${ }^{19}$ The delivery of second doses of COVID-19 vaccinations in care homes may be lower because it was difficult to follow-up on individuals who were not vaccinated or partially vaccinated and moved into a care home after or between mobile vaccination unit visits. It is important to further investigate the increased odds of being unvaccinated with dose 2 in care homes, as residents have been disproportionately affected by SARS-CoV-2. ${ }^{20}$ It is unlikely that the death rates are associated with low coverage because deaths are recorded in a timely manner in the NIMS ${ }^{21}$ although it is possible that care home residents might have had COVID-19 or another illness at the time of offer, thus causing a lag in the number of second doses received.

Both the highest odds and the predictive margins for being unvaccinated for dose 1 among all individuals in the study and for dose 2 among those aged 70 years and above were observed in the most deprived and among the black/African/Caribbean ethnicity. This coincides with findings assessing COVID-19 vaccine coverage for those aged 70 years and above using the 2011 Office for National Statistics (ONS) denominator population estimates ${ }^{22}$ and using general practice records for population estimates based on registrations for two of the three GP IT System Suppliers in England..$^{23}$ Furthermore, among those aged 70 years and above, the odds and prevalence of being unvaccinated among those of Pakistani ethnicity further increased for dose 2.

Our findings highlight the structural and complex interplay of ethnicity and deprivation, which has also been observed with findings on SARS-CoV-2 infection, hospitalisations and mortality. ${ }^{24}{ }^{25}$ This also concurs with a study assessing households in the UK that found individuals of an ethnic minority and a lower socioeconomic status were associated with greater COVID-19 vaccine hesitancy. ${ }^{26}$

\section{Strengths and limitations of this study}

Our study has several strengths. This is the first study assessing characteristics associated with COVID-19 vaccine coverage for all individuals aged 50 years and above in England, and one of the first studies globally assessing COVID-19 vaccine coverage. Our study also uses data from the NIMS, which is likely to be more complete than other datasets used to estimate COVID-19 vaccine coverage because it is based on all individuals in England with a registered NHS number. Furthermore, immunisation registers have proven to be fundamental when assessing and protecting the population, can be used for linkage to health-outcome databases and can play a key role in the delivery of a national immunisation programme. ${ }^{27-29}$ This is the first time England has developed a centralised national system capturing individuallevel data for both vaccination status and demographic characteristics. Previous studies assessing factors influencing vaccine coverage in England have been based on aggregate general practice-level data, in which estimates such as deprivation were based on the general practice postcode. Having individual-level data for frontline healthcare workers, care home residents and others allowed us to link individual NHS numbers to properly account for factors relating to these individuals. Such granular data are not available in similar studies or in general practice records.

We are unable to capture details on the total number of individuals without an NHS number and, of those who had not received a vaccine. Therefore, it is possible we have underestimated the number of vaccines delivered and odds of being unvaccinated for characteristics such as ethnic groups in which we have seen the greatest impact. The proportion of individuals aged 50 years and above with no NHS number is expected to be marginal. The NIMS population estimates are larger compared with the 2020 ONS mid-year population estimates (online supplemental figure 1), thus possibly overestimating population estimates. However, ONS estimates are based on the 2011 census and do not take into account any changes or movements throughout the pandemic, which may be reflected in NHS records. ${ }^{30}$ Furthermore, it is possible that there could be residual errors in data entry on the point-of-care applications at the vaccination sites, although these errors 
are not widespread. Although the NIMS was rapidly set up for monitoring COVID-19 vaccinations, it was piloted with influenza vaccinations delivered in the 2020/21 influenza season and the trends observed in our study align with other immunisation programmes.

\section{CONCLUSIONS}

This study provides evidence that in England, being male, being in a younger age group, belonging to certain minority groups, living in urban setting or being a care home resident were associated with low COVID-19 vaccine coverage. The largest odds of being unvaccinated was observed among those of black/African/Caribbean ethnicity and those in the most deprived decile. It is of utmost importance to reduce inequalities in vaccine coverage, particularly among black, Asian and minority ethnic groups, and care home residents who have been most impacted by the SARS-CoV-2 infection. The delivery of the COVID-19 vaccination programme should continue to be investigated. Delivery strategies, such as the use of mass immunisation sites and mobile vaccination units for populations where vaccine coverage is lower, should be evaluated to determine whether it could be applied to routine immunisations. As vaccine coverage increases in England, tailored strategies that consider barriers specific to undervaccinated groups, such as vaccine hesitancy, ${ }^{31}$ should be designed and implemented to improve COVID-19 vaccine coverage.

\section{Author affiliations}

${ }^{1}$ Immunisation and Countermeasures Division, Public Health England, London, UK

${ }^{2}$ Vaccines and Countermeasures Division, Public Health England, London, UK

${ }^{3}$ Health Intelligence Division, Health Improvement Directorate, Public Health England, York, UK

${ }^{4}$ Faculty of Medicine, Bar-llan University, Safed, Israel

Acknowledgements We would like to thank the Public Health England COVID-19 Data Science and ImmForm Teams, NHS England, NHS Digital and System C for their roles in developing and managing vaccination systems and datasets.

Contributors ET is the guarantor of the paper and confirms that the manuscript is honest, accurate and transparent. There are no important aspects of the paper omitted. ET, JS, CNJC, MR, JL-B, ME and JW provided overview of the project concept. ET, JS, YR, CT, CNJC, ME, JW, PR worked on the study design. AM, HH, $T R, E C, C T, A L, E T, Y R, J S$ and SL worked on acquiring and preparing the data for analysis. ET and NA prepared the statistical analyses and interpretation of the data. ET and JS prepared the manuscript and all authors reviewed the manuscript.

Funding Funding from Public Health England for the submitted work.

Map disclaimer The inclusion of any map (including the depiction of any boundaries therein), or of any geographic or locational reference, does not imply the expression of any opinion whatsoever on the part of BMJ concerning the legal status of any country, territory, jurisdiction or area or of its authorities. Any such expression remains solely that of the relevant source and is not endorsed by BMJ. Maps are provided without any warranty of any kind, either express or implied.

Competing interests None declared.

Patient consent for publication Not applicable.

Ethics approval Surveillance of COVID-19 vaccination data is undertaken under Regulation 3 of The Health Service (Control of Patient Information) Regulations 2002 to collect confidential patient information (www.legislation.gov.uk/uksi/2002/1438/ regulation/3/made) under Sections 3(i) (a) to (c), 3(i) (d) (i) and (ii) and 3 (3).

Provenance and peer review Not commissioned; externally peer reviewed.
Data availability statement No data are available. This work is carried out under Regulation 3 of The Health Service (Control of Patient Information) (Secretary of State for Health, 2002) (3) using patient identification information without individual patient consent. Data cannot be made publicly available for ethical and legal reasons, that is, public availability would compromise patient confidentiality as data tables list single counts of individuals rather than aggregated data.

Supplemental material This content has been supplied by the author(s). It has not been vetted by BMJ Publishing Group Limited (BMJ) and may not have been peer-reviewed. Any opinions or recommendations discussed are solely those of the author(s) and are not endorsed by BMJ. BMJ disclaims all liability and responsibility arising from any reliance placed on the content. Where the content includes any translated material, BMJ does not warrant the accuracy and reliability of the translations (including but not limited to local regulations, clinical guidelines, terminology, drug names and drug dosages), and is not responsible for any error and/or omissions arising from translation and adaptation or otherwise.

Open access This is an open access article distributed in accordance with the Creative Commons Attribution Non Commercial (CC BY-NC 4.0) license, which permits others to distribute, remix, adapt, build upon this work non-commercially, and license their derivative works on different terms, provided the original work is properly cited, appropriate credit is given, any changes made indicated, and the use is non-commercial. See: http://creativecommons.org/licenses/by-nc/4.0/.

\section{ORCID iDs}

Elise Tessier http://orcid.org/0000-0002-2712-7971

Michael Edelstein http://orcid.org/0000-0002-7323-0806

Mary Ramsay http://orcid.org/0000-0002-7156-7640

Jamie Lopez-Bernal http://orcid.org/0000-0002-1301-5653

Nick Andrews http://orcid.org/0000-0003-2069-2684

Julia Stowe http://orcid.org/0000-0002-3999-6461

\section{REFERENCES}

1 Baraniuk C. Covid-19: how the UK vaccine rollout delivered success, so far. BMJ 2021;372:n421.

2 Department of Health and Social Care. Statement from the UK chief medical officers on the prioritisation of first doses of COVID-19 vaccines, 2020 2021. Available: https://www.gov.uk/government/ news/statement-from-the-uk-chief-medical-officers-on-theprioritisation-of-first-doses-of-covid-19-vaccines [Accessed $02 \mathrm{Feb}$ 2021].

3 Public Health England. COVID-19 - SARS-CoV-2 Chapter 14a, 2021. Available: https://www.gov.uk/government/publications/covid-19the-green-book-chapter-14a

4 Public Health England. National protocol for COVID-19 vaccine Moderna, 2021. Available: https://www.gov.uk/government/ publications/national-protocol-for-covid-19-vaccine-moderna [Accessed 25 May 2021].

5 Department of Health and Social Care. Optimising the COVID-19 vaccination programme for maximum short-term impact, 20212021. Available: https://www.gov.uk/government/publications/prioritisingthe-first-covid-19-vaccine-dose-jcvi-statement/optimising-thecovid-19-vaccination-programme-for-maximum-short-term-impact [Accessed 03 Feb 2021].

6 Care. DoHS. Joint Committee on vaccination and immunisation: advice on priority groups for COVID-19 vaccination, 2020. Available: https://www.gov.uk/government/publications/ priority-groups-for-coronavirus-covid-19-vaccination-advicefrom-the-jcvi-30-december-2020/joint-committee-onvaccination-and-immunisation-advice-on-priority-groups-for-covid19-vaccination-30-december-2020 [Accessed 20 May 2021].

7 NHS England. Who is at high risk from coronavirus (COVID-19), 2021. Available: https://www.nhs.uk/conditions/coronavirus-covid19/people-at-higher-risk/who-is-at-high-risk-from-coronavirus/ [Accessed 21/07/2021].

8 NHS Digital. COVID-19 vaccinations, 2021. Available: https:// www.england.nhs.uk/statistics/statistical-work-areas/covid-19vaccinations/ [Accessed 18 Jun 2021].

9 Ward C, Byrne L, White JM, et al. Sociodemographic predictors of variation in coverage of the National shingles vaccination programme in England, 2014/15. Vaccine 2017;35:2372-8.

10 Tessier E, Warburton F, Tsang C, et al. Population-Level factors predicting variation in influenza vaccine uptake among adults and young children in England, 2015/16 and 2016/17. Vaccine 2018;36:3231-8. 
11 Green HK, Andrews N, Letley L, et al. Phased introduction of a universal childhood influenza vaccination programme in England: population-level factors predicting variation in national uptake during the first year, 2013/14. Vaccine 2015;33:2620-8.

12 Public Health England. Flu and flu vaccination 2019/20: a toolkit for care homes (South West, 2020.

13 NHS Digital. Nhs spine, 2021. Available: https://digital.nhs.uk/ services/spine [Accessed 27 May 2021].

14 NHS England. Nhs England invites everyone aged 50 and over to be jabbed as NHS vaccination programme marks 100th day, 2021. Available: https://www.england.nhs.uk/2021/03/nhsengland-invites-everyone-aged-50-and-over-to-be-jabbed-asnhs-vaccination-programme-marks-100th-day/ [Accessed 05 Jun 2021].

15 NHS England. Nhs invites people aged 56 to 59 for their COVID Jab, 2021. Available: https://www.england.nhs.uk/2021/03/56-59/ [Accessed 05 Jun 2021].

16 NHS Digital. Vaccination of JCVI cohorts 5-6 and additional funding for vaccination in residential settings, 2021. Available: https://www. england.nhs.uk/coronavirus/publication/vaccination-of-jcvi-cohorts5-6-and-additional-funding-for-vaccination-in-residential-settings/ [Accessed 07 Jun 2021].

17 Bell S, Clarke R, Mounier-Jack S, et al. Parents' and guardians' views on the acceptability of a future COVID-19 vaccine: a multi-methods study in England. Vaccine 2020;38:7789-98.

18 Public Health England. Seasonal influenza vaccine uptake in GP patients: winter season 2020 to 2021, 2021. Available: https://assets. publishing.service.gov.uk/government/uploads/system/uploads/ attachment_data/file/996033/Annual-Report_SeasonalFlu-Vaccine GPs_2020_to_2021.pdf

19 Mills MC, Salisbury D. The challenges of distributing COVID-19 vaccinations. EClinicalMedicine 2021;31:100674.

20 Gordon AL, Goodman C, Achterberg W, et al. Commentary: COVID in care homes-challenges and dilemmas in healthcare delivery. Age Ageing 2020;49:701-5.
21 NHS Digital. Mortality data review, 2020. Available: https://digital.nhs. uk/coronavirus/coronavirus-data-services-updates/mortality-datareview [Accessed 22 Jun 2021].

22 Statistics. OfN. Coronavirus and vaccination rates in people aged 70 years and over by sociodemographic characteristic, England: 8 December 2020 to 9 may 20212021.

23 Curtis HJ, Inglesby P, Morton CE. Trends and clinical characteristics of COVID-19 vaccine recipients: a federated analysis of 57.9 million patients' primary care records in situ using OpenSAFELY. medRxiv2021:2021.01.25.21250356.

24 Mathur R, Rentsch CT, Morton CE, et al. Ethnic differences in SARSCoV-2 infection and COVID-19-related hospitalisation, intensive care unit admission, and death in 17 million adults in England: an observational cohort study using the OpenSAFELY platform. Lancet 2021;397:1711-24

25 Aldridge RW, Lewer D, Katikireddi SV, et al. Black, Asian and minority ethnic groups in England are at increased risk of death from COVID-19: indirect standardisation of NHS mortality data. Wellcome Open Res 2020;5:88.

26 Robertson E, Reeve KS, Niedzwiedz CL, et al. Predictors of COVID-19 vaccine hesitancy in the UK household longitudinal study. Brain Behav Immun 2021;94:41-50.

27 Crowcroft NS, Levy-Bruhl D. Registries: an essential tool for maximising the health benefits of immunisation in the 21 st century. 2017;22:30523.

28 Johansen K, Lopalco PL, Giesecke J. Immunisation registers-important for vaccinated individuals, vaccinators and public health. Euro Surveill 2012;17:20151.

29 Pebody R. Vaccine registers - experiences from Europe and elsewhere. 2012;17:20159.

30 UK Health Security Agency Transparency and data - UKHSA's vaccines report, 2021. Available: https://ukhsa.blog.gov.uk/2021/11/ 02/transparency-and-data-ukhsas-vaccines-report/

31 Freeman D, Loe BS, Chadwick A, et al. COVID-19 vaccine hesitancy in the UK: the Oxford coronavirus explanations, attitudes, and narratives survey (Oceans) II. Psychol Med 2020:1-15. 